\title{
EXACT SOLUTIONS TO THE COAGULATION EQUATION
}

\author{
M.H. ERNST and E.M. HENDRIKS \\ Instituut voor Theoretische Fysica, Rijksuniversiteit Utrecht, The Netherlands 1 \\ and
}

R.M. ZIFF

Department of Mechanical Engineering, State University of New York, Stony Brook, NY 11794, USA

Received 31 August 1982

Explicit post-gelation solutions are presented for Smoluchowski's coagulation equation with factorizable transition kernels $K_{i j}=s_{i} s_{j}$, when $s_{k}=k^{\omega}(\omega>1 / 2)$ and $s_{k}=\exp [\alpha(k-1)](\alpha>0)$. In such solutions the total mass of sol (finite clusters) is not conserved in time, as the sol is loosing mass to the gel (infinite cluster). For the kernels $K_{i j}=i \mu^{\nu}+j \mu_{i} \nu(\mu$ $=0,1, v$ general) Smoluchowski's equation can be solved sequentially in terms of a transformed time variable.

In coagulation processes Smoluchowski's equation describes the time evolution of the size distribution function $c_{k}(t)$, denoting the concentration of clusters of size $k$ :

$\dot{c}_{k}=\frac{1}{2} \sum_{i+j=k} K_{i j} c_{i} c_{j}-c_{k} \sum_{j=1}^{\infty} K_{k j} c_{j}$,

where the coagulation kernel $K_{i j}$ models the coalescence mechanism of an $i$ - and a $j$-cluster. Here we neglect fragmentation, source and sedimentation terms which are frequently added in studies of aerosol coagulation.

The exact solution for a given initial distribution $c_{k}(0)$ is only known for kernels of the general form $K_{i j}=A+B(i+j)+C_{i j}[1-4]$. If $C \neq 0$, the solution $c_{k}(t)$ undergoes a phase transition, called gelation; viz. before the gelpoint $t_{\mathrm{c}}$ the total mass of finite size clusters (sol), $M_{1}(t)=\Sigma k c_{k}(t)$, is constant; at $t_{\mathrm{c}}$ an infinite cluster (gel) appears causing $M_{1}(t)$ to decrease for $t \geqslant t_{\mathrm{c}}$, as the sol is loosing mass to the gel.

The purpose of the present letter is to show (i) that explicit post-gelation solutions, with $\dot{M}_{1}(t) \neq 0$, can

1 Mailing address: Princetonplein 5, P.O. Box 80006, 3508 TA Utrecht, The Netherlands. be found for factorizable kernels $K_{i j}=s_{i} s_{j}$ under some further restrictions on $s_{k}$, and (ii) that for a few other kernels the coagulation equation can be solved sequentially. Details will be published elsewhere [5].

A special post-gelation solution of the coagulation equation is suggested by the exact solution for a monodisperse initial distribution in the cases $s_{k}=(f-2) k$ +2 with $f \geqslant 3$ [2], and $s_{k}=k$ [3]. Past the gel point the size distribution is simply given by:

$c_{k}(t)=c_{k}\left(t_{\mathrm{c}}\right)\left[1+b\left(t-t_{\mathrm{c}}\right)\right]^{-1} \quad\left(t \geqslant t_{\mathrm{c}}\right)$.

In the former case $t_{\mathrm{c}}=1 / f(f-2)$ and $b=f^{2}(f-2) /$ $(f-1)$ and in the latter case $t_{\mathrm{c}}=b=1$. Up to the gel point the mass is conserved $\left(M_{1}=1\right)$, and past the gel point $M_{1}(t)=\left[1+b\left(t-t_{\mathrm{c}}\right)\right]^{-1}$. Therefore we investigate whether a post-gelation solution of the form (2) [satisfying $M_{1}\left(t_{\mathrm{c}}\right)=1$ ] is possible for the models $K_{i j}$ $=s_{i} s_{j}$. On substituting (2) into (1) we find that the time part cancels, and we obtain a recursion relation for $c_{k}\left(t_{\mathrm{c}}\right)$, where consistency for $k=1$ requires $b$ $=\Sigma s_{k} c_{k}\left(t_{\mathrm{c}}\right)$ (a proper choice of time unit gives $s_{1}=1$ ). By introducing $n_{k}=c_{k}\left(t_{\mathrm{c}}\right) / b$ it takes the simple form:

$\left(s_{k}-1\right) n_{k}=\frac{1}{2} \sum_{i+j=k} s_{i} s_{j} n_{i} n_{j}$,

which has to be solved subject to the condition: 
$\sum_{s_{k} n_{k}=1, \quad \text { or }} \sum n_{k}=\frac{1}{2}$.

For a given $n_{1},(3)$ determines all $n_{2}, n_{3}, \ldots$. Choosing $n_{1}$ such that (4) is satisfied, one can then in principle find $b$ from $M_{1}\left(t_{\mathrm{c}}\right)=1$ through $b^{-1}=\Sigma k n_{k}$. The solution will be obtained by introducing generating functions:

$G(x)=\sum_{k} n_{k} \mathrm{e}^{k x}, \quad F(x)=\sum_{k} s_{k} n_{k} \mathrm{e}^{k x}$.

If $1 / s_{k}$ can be written as a Laplace transform, i.e.

$1 / s_{k}=\int_{0}^{\infty} \mathrm{d} y \sigma(y) \exp (-k y)$,

then $F$ and $G$ are related by

$G(x)=\int_{0}^{\infty} \mathrm{d} y \sigma(y) F(x-y)$,

and it follows from the recursion relation (3) that

$F-G=\frac{1}{2} F^{2}, \quad$ or $F=1-(1-2 G)^{1 / 2}$,

or

$F(x)-\frac{1}{2} F^{2}(x)=\int_{0}^{\infty} \mathrm{d} y \sigma(y) F(x-y)$.

We discuss two classes of models: $s_{k}=k^{\omega}$, where $\sigma(y)$ $=y^{\omega-1} / \Gamma(\omega)[$ when $\omega$ is a positive integer $F$ and $G$ are simply related by differentiation: $\left.F=(\mathrm{d} / \mathrm{d} x)^{\omega} G\right)$, and $s_{k}=\exp [\alpha(k-1)]$, where $\sigma(y)=\mathrm{e}^{\alpha} \delta(y-\alpha)$.

In the case, $s_{k}=k^{2}$, where $F=G^{\prime \prime}=\Sigma k^{2} n_{k} \mathrm{e}^{k x}$, (6a) reduces to a differential equation, which can be solved to yield:

$\mathrm{e}^{x}=\frac{(\sqrt{3}+1)(\sqrt{3}-r)}{(\sqrt{3}-1)(\sqrt{3}+r)} \exp [\sqrt{3}(r-1)]$,

where $r=\sqrt{3-2 F}$. The $n_{k}$ follow by expanding $F(x)$ in powers of $\mathrm{e}^{x}$, using Lagrange's expansion [6]. $n_{1}$ is obtained as:

$n_{1}=\lim _{x \rightarrow-\infty} \mathrm{e}^{-x} F(x)=6(2-\sqrt{3}) \mathrm{e}^{\sqrt{3}-3}$,

and the higher $n_{k}$ are: $n_{k}=n_{1}^{k} \sum_{l=0}^{k-1} \frac{(k+l+1)(k-1) ! k^{k-3-l}}{l !(k-l+1) !(k-l-1) !}\left(\frac{-1}{6}\right)^{l}$.

From $G^{\prime}(0)=b^{-1}$ we obtain $b=M_{2}\left(t_{\mathrm{c}}\right)=\sqrt{3}$. The large- $k$ dependence of this solution is:

$n_{k} \sim(2 \pi \sqrt{3})^{-1 / 2}\left[k^{-7 / 2}+(3 \sqrt{3} / 8) k^{-9 / 2}+\ldots\right]$.

Thus we have determined all parameters (except $t_{\mathrm{c}}$ ) in the post-gelation solution (2) for the model $s_{k}=k^{2}$, and obtained what constitutes a new solution to the coagulation equation.

In the general case $s_{k}=k^{\omega}$, post-gelation solutions only exist for $\omega>1 / 2[7,8]$, and the quantities $b$ and $n_{1}$ have been determined numerically [5]. Analytically only a few limiting properties can be calculated: viz.

(i) the small-x behavior of $F$ is $F(x) \approx 1-(-2 x / b)^{1 / 2}$ $+\ldots$, implying $n_{k} \sim(2 \pi b)^{-1 / 2} k^{-\omega-3 / 2}+\ldots$ at large $k$, and (ii) for large $\omega$

$k^{\omega} n_{k} \approx-\left(-\frac{1}{2}\right)_{k} / k !+\frac{1}{4} 2^{-\omega}(-3 / 2)_{k} /[3(k-1) !]$,

$b \approx\left(\sum_{k n_{k}}\right)^{-1} \approx 2-\frac{1}{2} 2^{-\omega}+\ldots$,

$\sum k^{\alpha} n_{k} \approx \frac{1}{2}+1 / 8\left(2^{\alpha}-1\right) 2^{-\omega}+\ldots \quad(\alpha \ll \omega)$

where $(a)_{k}=\Gamma(a+k) / \Gamma(k)$.

In the case $s_{k}=\exp [\alpha(k-1)]$ post-gelation solutions only exist for $\alpha>0$. The integral equation (6b) reduces to a difference equation:

$F(x)-\frac{1}{2} F^{2}(x)=\mathrm{e}^{\alpha} F(x-\alpha)$,

to be solved subject to $F(0)=1$. The relevant quantities can be determined as follows:

$\lim _{x \rightarrow-\infty} \mathrm{e}^{-x} F(x)=n_{1}$,

and all $n_{k}$ are calculated from (3). Since $F(0)=\Sigma s_{k} n_{k}$ $<\infty$, all moments exist, and

$b^{-1}=\mathrm{e}^{\alpha} F^{\prime}(-\alpha) ; \quad \sum k^{l} n_{k}=\mathrm{e}^{\alpha} F^{(l)}(-\alpha)$,

where $F^{(l)}(x)$ denotes the $l$ th derivative. The solution is determined by defining numbers $p_{k}=\mathrm{e}^{k \alpha} F(-k \alpha)$, which obey the recursion relation

$p_{k+1}=p_{k}\left(1-\frac{1}{2} \mathrm{e}^{-k \alpha} p_{k}\right) \quad\left(p_{0}=1\right)$. 
The limiting value gives $p_{\infty}=n_{1}$. For $\alpha>0$ the $p_{k}$ constitute a monotonically decreasing sequence of positive numbers, implying the existence of $p_{\infty}$, and the post-gelation solution (2) exists. (For $\alpha \leqslant 0$ no post-gelation solutions are possible). If $\alpha$ is not too small, (14) has the solution:

$p_{\infty}=\frac{1}{2}\left(1-\frac{1}{4} \mathrm{e}^{-\alpha}-\frac{1}{4} \mathrm{e}^{-2 \alpha}-\mathrm{e}^{-3 \alpha} / 8+\ldots\right)$,

and the $n_{k}$ can be determined as a power series in $\mathrm{e}^{-\alpha}$.

To obtain $b$ we introduce another set of numbers $q_{k}=\mathrm{e}^{k \alpha} F^{\prime}(-\alpha)$, where $q_{\infty}=p_{\infty}=n_{1}$. A recursion relation for $q_{k}$ is obtained by differentiating (12):

$q_{k+1}=q_{k}\left(1-\mathrm{e}^{-k \alpha} p_{k}\right) \quad\left(q_{\infty}=n_{1}\right)$,

so that

$b=\left(q_{1}\right)^{-1}=\left(n_{1}\right)^{-1} \prod_{k=1}^{\infty}\left(1-\mathrm{e}^{-k \alpha} p_{k}\right)$.

In general the recursion relations (14) and (16) must be solved numerically, yielding $n_{1}$ and $b$ as a function of the parameter $\alpha$ [5]. The large- $k$ behavior is given by

$n_{k} \sim(2 \pi b)^{-1 / 2} k^{-3 / 2} \exp [(1-k) \alpha]+\ldots$.

The limiting case $\alpha \gg 1$ is completely analogous to the large- $\omega$ case, and the corresponding results are obtained from (11) by replacing $k^{\omega} n_{k}$ on the first line by $\mathrm{e}^{(k-1) \alpha} n_{k}$, and $2^{-\omega}$ everywhere by $\mathrm{e}^{-\alpha}$.

The question arises $[5,8]$ whether the special solution (2) evolves from a monodisperse initial distribution, as is the case in the exactly solvable models. Although the solution to the initial value problem is not known, we can give a definite and negative answer to this question for the cases: $s_{k}=k^{\omega}$ with $\omega \gtrsim 1.1$ and $s_{k}=\exp [\alpha(k-1)]$ for $\alpha>\log 2$ by using inequalities. For the remaining range of $\alpha$ - and $\omega$-values (except $\omega$ $=1$ ) we expect also a negative answer, but the question has not been settled.

In the second part of this letter we discuss the models $K_{i j}=i^{\mu}+j i^{\mu}$ and $K_{i j}=i^{\lambda}+j^{\lambda}$, for which the coagulation equation can be solved sequentially. As far as gelation is concerned the following is known about these models. If $K_{i j} \leqslant C(i+j)$ (here $\mu \leqslant 0$ and $\lambda \leqslant 1$ ), White [9] has shown that the coagulation equation does not show a gelation transition. If $\mu>0$ and $\lambda>1$ gelation occurs [10]; possible pre-gelation solutions $\left(t<t_{\mathrm{c}}\right)$ have the scaling form $c_{k}(t) \sim k^{-\tau} \Phi\left(k\left(t_{\mathrm{c}}-t\right)^{1 / \sigma}\right)$

as $t \uparrow t_{\mathrm{c}}$ and $k \rightarrow \infty$ with $x=k\left(t_{\mathrm{c}}-t\right)^{1 / \sigma}$ fixed; and possible post-gelation solutions have the form $c_{k}(t)$ $\sim k^{-\tau} A(t)$ for large $k$. In the first model with $\mu>0$ (where existence of global solutions follows from the work of Leyvraz and Tschudi [8] for $\mu \leqslant 1$ ) we found $\tau=2+\frac{1}{2} \mu$ and $\tau+\sigma=\mu+2$. The second model with $\lambda>1$ is only meaningful in the pre-gelation stage, where the exponents $\sigma$ and $\tau$ in the scaling form are related as $\sigma+\tau=\lambda+1$. At a finite time $t_{\mathrm{c}}$ the system undergoes a gelation transition. Post-gelation solutions do not exist, since the coagulation equation is no longer well-defined beyond the gel point, as it contains divergent quantities.

The coagulation equation for the first model $K_{i j}$ $=i j^{\mu}+j i^{\mu}$ reads:

$\dot{c}_{k}=\sum_{i+j=k} i j^{\mu} c_{i} c_{j}-k c_{k} M_{\mu}-k^{\mu} c_{k} M_{1}$,

where $M_{\alpha} \equiv \Sigma k^{\alpha} c_{k}$. It has been solved by Lushnikov and Piskunov [11] for $\mu<0$, where $M_{1}(t)=1$ for all $t \geqslant 0$. Their method can be trivially extended to obtain pre-gelation solutions for the case $\mu>0$ (where $M_{1}(t)=1$ for $\left.t \leqslant t_{\mathrm{c}}\right)$. With the help of the substitution $c_{k}(t)=\nu_{k}(t) \exp \left[k M_{0}(t)\right]$

and the moment equation $\dot{M}_{0}=-M_{\mu}$ (valid in the pregelation stage), the coagulation equation can be transformed into:

$\dot{\nu}_{k}=\sum_{i+j=k} i j^{\mu} \nu_{i} \nu_{j}-k^{\mu} \nu_{k}$.

The solution of (19) is given in ref. [11], where the unknown $M_{0}(t)$ can be determined from the transcendental equation:

$1=\sum_{k c_{k}}=\sum_{k \nu_{k}} \exp \left[k M_{0}(t)\right]$.

The above substitution does not lead to any simplifications in the post-gelation stage, neither does it enable us to locate the gel point exactly. However, in the range $0<\mu \leqslant 1$ we have found $[5,10]$ the lower bound $t_{\mathrm{b}}=\left[2 \mu M_{2}^{\mu}(0)\right]^{-1}$ for $t_{\mathrm{c}}$. For $\mu \geqslant 1$ the lowerbound changes into an upperbound.

The coagulation equation for the second model $K_{i j}=i^{\lambda}+j^{\lambda}$, reading: 
$\dot{c}_{k}=\sum_{i+j=k} i^{\lambda} c_{i} c_{j}-c_{k} M_{\lambda}-k^{\lambda} c_{k} M_{0}$,

can also be solved sequentially, as follows: we introduce new variables by the transformation (reminiscent of Lushnikov's transformation [12] where $M_{0}(t)$ is replaced by $\left.c_{1}(t)\right)$ :

$\tau=\int_{0}^{t} \mathrm{~d} t^{\prime} M_{0}\left(t^{\prime}\right) ; \quad \nu_{k}(\tau)=c_{k}(t) / M_{0}(t)$

and with the help of the moment equation $\dot{M}_{0}$

$=-M_{0} M_{\lambda}$ we transform (20) into:

$\mathrm{d} \nu_{k}(\tau) / \mathrm{d} \tau=\sum_{i+j=k} i^{\lambda} \nu_{i} \nu_{j}-k^{\lambda} \nu_{k}$,

to be solved subject to the condition, $\Sigma \nu_{k}=1$. Once all $\nu_{k}(\tau)$ have been determined the function $M_{0}(t)$ $\equiv \mu_{0}(\tau)$ can be found as a function of $\tau$ from $\Sigma k \nu_{k}$ $=1 / \mu_{0}(\tau)=1 / M_{0}(t)$ and the original $t$-variable can be recovered by differentiating $\tau(t)$ in (21) and solving for $t$ with the result

$t=\int_{0}^{\tau} \mathrm{d} \tau^{\prime}\left[\mu_{0}\left(\tau^{\prime}\right)\right]^{-1}$

For a monodisperse initial distribution where $\nu_{k}(0)$ $=\delta_{k 1}$, the first few $v_{k}$ read explicitly:

$\nu_{1}(\tau)=\mathrm{e}^{-\tau}$,

$\nu_{2}(\tau)=(2 \omega-2)^{-1}[\exp (-2 \tau)-\exp (-2 \omega \tau)]$,

$\nu_{3}(\tau)=-\left(2^{\omega}-2\right)^{-1}\left(3^{\omega}-2^{\omega}-1\right)^{-1}\left(2^{\omega}+1\right)$

$X\left\{\exp \left[-\left(2^{\omega}+1\right) \tau\right]-\exp \left(-3^{\omega} \tau\right)\right\}+\left(2^{\omega}-2\right)^{-1}$

$\times\left(3^{\omega}-3\right)^{-1}\left(2^{\omega}+1\right)[\exp (-3 \tau)-\exp (-3 \omega \tau)]$.
For the case $\lambda \leqslant 1$ the present method can be used for all $t$. For the case $\lambda>1$ the kinetic equation is only well-defined below the gel point. We have not been able to determine $t_{\mathrm{c}}$ exactly, but only obtained the bound

$t_{\mathrm{c}} \leqslant\left[2(\lambda-1) M_{2}^{\lambda-1}(0)\right]^{-1}$.

Furthermore, without an intervening gelation transition, $M_{0}(t)$ would become negative within a finite time $t_{0}$, obeying $t_{\mathrm{c}}<t_{0} \leqslant\left[M_{0}(0)\right]^{\lambda-1} /(\lambda-1)$, so that there would not exist a one-to-one relationship between $t$ and $\tau$.

This work was supported in part by the Office of Basic Energy Sciences, US Department of Energy.

\section{References}

[1] R.L. Drake, in: Topics in current aerosol research, Vol. 3, eds. G.M. Hidy and J.R. Brock (Pergamon, New York, 1972) Pt. 2.

[2] R.M. Ziff and G. Stell, J. Chem. Phys. 73 (1980) 3492.

[3] F. Leyvraz and H.R. Tschudi, J. Phys. Al4 (1982) 3389.

[4] R.J. Cohen and G.B. Benedek, J. Chem. Phys, to be published.

[5] E.M. Hendriks, M.H. Ernst and R.M. Ziff, J. Stat. Phys., to be published.

[6] M. Abramowitz and I.A. Stegun, Handbook of mathematical functions (Dover Publications, New York).

[7] R.M. Ziff, E.M. Hendriks and M.H. Ernst, Phys. Rev. Lett. 49 (1982) 593.

[8] F. Leyvraz and H.R. Tschudi, J. Phys. Al5 (1982) 1951.

[9] W.W. White, Proc. Am. Math. Soc. 80 (1980) 273.

[10] M.H. Ernst, E.M. Hendriks and R.M. Ziff, J. Phys. A, to be published.

[11] A.A. Lushnikov and V.N. Piskunov, translated from Kolloidn. Zh. 37 (1975) 285 .

[12] A.A. Lushnikov, J. Colloid Interface Sci. 45 (1973) 549. 